\title{
On some properties of $\gamma$-semi- $R_{0}$ spaces
}

\section{SABIR HUSSAIN}

\section{ABSTRACT.}

The purpose of this paper is to introduce $\gamma$-semi- $R_{0}$ spaces in terms of $\gamma$-semi-open sets which contains the $\gamma$-semi-closure of each of its singletons. Some properties of $\gamma$-semi- $R_{0}$ spaces are also discussed and explored.

\section{REFERENCES}

[1] Ahmad, B. and Hussain, S., Properties of $\gamma$-Operations on Topological Spaces, Aligarh Bull. Math., 22 (2003), No. 1, 45-51

[2] Ahmad, B. and Hussain, S., $\gamma$-Semi-Open Sets in Topological Spaces II, Sotheast Asian Bull. Math., 34 (2010), No. 5, 997-1008

[3] Ahmad, B. and Hussain, S., $\gamma$-Convergence in Topological Spaces, Southeast Asian Bull. Math., 29 (2005), $832-842$

[4] Ahmad, B. and Hussain, S., $\gamma^{*}$-Regular and $\gamma$-Normal Space, Math. Today., 22 (2006), No. 1, 37-44

[5] Ahmad, B. and Hussain, S., On $\gamma$-s-Closed Subspaces, Far East Jr. Math. Sci., 31 (2008), No. 2, 279-291

[6] Ahmad, B., Hussain, S. and Noiri, T., On Some Mappings in Topological Space, Eur. J. Pure Appl. Math., 1 (2008), 22-29

[7] Ahmad, B., Hussain, S. and Noiri, T., $\gamma$-Semi-Open Sets in Topological Spaces, Asian Eur. J. Math., 3 (2010), No. 3, 427-433. DOI: 10.1142/S1793557110000337

[8] Ahmad, B., Hussain, S. and Noiri, T., On $\gamma$-Semi Continuous Functions, Punjab University Jr. Math., 42 (2010), 57-65

[9] Hussain, S. and Ahmad, B., Applications of Minimal $\gamma$-Semi-Open Sets , Fasciculli Mathematici, 46 (2011), 65-76

[10] Hussain, S. and Ahmad, B., On $\gamma$-s-Closed Spaces, Sci. Magna Jr., 3 (2007), No. 4, 89-93

[11] Hussain, S. and Ahmad, B., On $\gamma$-s-Regular Spaces and Almost $\gamma$-s-Continuous Functions, Lobackevskii. J. Math., 30(2009), No. 4, 263-268. DOI: $10.1134 / 51995080209040039$

[12] Kasahara, S., Operation-Compact Spaces, Math. Japon., 24 (1979), 97-105

[13] Ogata, H., Operations on Topological Spaces and Associated Topology, Math. Japon., 36 (1991), No. 1, 175-184

DePARTMENT OF MATHEMATics

COLLEGE OF SCIENCE

QASSIM UNIVERSITY

P. O. BOX 6644, BURAYDAH 51452, SAUdi ARABIA

E-mail address: sabiriub@yahoo.com; sh.hussainequ.edu.sa 